\title{
Acoustic temperature measurement in a full-scale rocket noise field
}

\author{
Jarom H. Giraud \\ Advisor: Dr. Kent L. Gee
}

Physics 492R Capstone Project Report

6-July-2009

\begin{abstract}
A one-micron diameter platinum wire resistance thermometer measures temperature fluctuations generated by propagating noise produced by a horizontally-fired, static GEM-60 solid rocket motor. A relationship between small amplitude acoustic pressure and acoustic temperature is derived and the data are compared with those calculated from pressure data recorded by a nearby $3.18 \mathrm{~mm}$ condenser microphone. The validity of taking acoustic temperature measurements in a rocket field is discussed, particularly the role of turbulence induced temperature fluctuations.
\end{abstract}


Copyright 2009 Jarom H. Giraud

Brigham Young University 


\section{Introduction}

In an effort to measure high-frequency temperature fluctuations in air, scientists in the 1960s and 70s worked on developing thin-wire resistance thermometers_capable of measuring temperature fluctuations at thousands of hertz. ${ }^{1,2,3}$ These thin wires were generally made from platinum, stretched thin by the Wollaston process. ${ }^{1}$ Resistance temperature devices, $\mathrm{RTDs}_{2}$ sense variations of resistance in the wire and these are interpreted as temperature fluctuations. In the fabrication of RTDs, platinum has been extensively used because of its near linear relationship between resistance and temperature. Today, platinum is so extensively used in RTDs that they are sometimes referred to as PTDs.

There are several similarities between the use of an RTD to measure high-frequency temperature fluctuations and thin-wire anemometry. However, it is important to note that, strictly speaking, obtaining thin-wire resistance thermometer data is not anemometry. Anemometry results in the measuring of wind speed and direction while our temperature probe intends only to measure temperature fluctuations. Readers familiar with thin-wire anemometry will recognize our temperature probe configuration to be nearly identical to that used in constant current anemometry (CCA). CCA is a method where a constant current, $I$, is run through a small wire and the resulting voltage, $V$, is recorded. Temperature fluctuations will affect the resistance, $R$, of the wire and by using Ohm's law, $V=I R$, one can relate the measured changes in voltage to the temperature fluctuations. While the temperature information is sufficient for a RTD, in 
CCA, these fluctuations would then related to wind speed using ideas such as heat transfer.

Work has been done to model and measure acoustic temperature fluctuations in a waveguide and in shock tubes, ${ }^{4,5,6}$ however, to the knowledge of this research team, no work has been done to take acoustic temperature measurements in a full-scale rocket noise field. The near-field of a rocket motor is ideally suited for acoustic temperature measurements because the temperature fluctuations are large and they occur at frequencies easily measured using a thin wire probe. This unique measurement gives insight to the impact of acoustic temperature fluctuations in CCA and also demonstrates the relationship between pressure and temperature in an adiabatic process.

\section{Theory}

It is necessary for this experiment to know the relationship between acoustic pressure and acoustic temperature to properly calibrate the temperature probe. This relationship needed to directly relate these two aforementioned quantities by variables easily obtained in the field. The following is a derivation used to arrive at a small signal relationship between acoustic pressure and acoustic temperature. While this relationship it is not purported to be new, the authors are unaware of it's previous derivation.

The total quantities of density, $\rho$, temperature, $T_{K}$, and pressure, $P$, in air can be defined as

$$
\begin{gathered}
\rho=\rho_{0}+\Delta \rho \\
T_{K}=T_{K 0}+\Delta T
\end{gathered}
$$




$$
P=P_{0}+\Delta p
$$

where the variables denoted by the subscript ' 0 ' represent the ambient/equilibrium values and the variables preceded by the delta are the acoustically induced fluctuations.

The equation of state ${ }^{7}$ defines a relationship between $T_{K}$ and $P$ for a gas such as air as

$$
P=\rho r T_{K}
$$

where $r$ is the specific gas constant and written in terms of $\rho$ becomes

$$
\rho=\frac{P}{r T_{K}}
$$

Substituting Eq. (5) into the perfect gas adiabat ${ }^{7}$

$$
\frac{P}{P_{0}}=\left(\frac{\rho}{\rho_{0}}\right)^{\gamma},
$$

where $\gamma$ is the ratio of specific heats, one obtains the expression

$$
\frac{P}{P_{0}}=\left(\frac{P}{\rho_{0} r T_{K}}\right)^{\gamma} .
$$

Now, if $r$ is rewritten in terms of the ambient/equilibrium values, one obtains

$$
r=\frac{P_{0}}{\rho_{0} T_{K 0}} .
$$

By substituting Eq. (8) into Eq.(7), the result can be solved for the total temperature.

$$
T_{K}=T_{K 0}\left(1+\frac{\Delta p}{P_{0}}\right)^{\frac{\gamma-1}{\gamma}} .
$$

Equation (9) is perfectly viable as a relationship between acoustic pressure and acoustic temperature if the total temperature Eq. (2) is employed. As a matter of academic interest, a first order binomial expansion can then be employed to find 


$$
T_{K}=T_{K 0}+T_{K 0}\left(\left(\frac{\gamma-1}{\gamma}\right) \frac{\Delta p}{P_{0}}\right)
$$

Remembering $T_{K}$ is a total quantity, we subtract $T_{K 0}$ from Eq. (10) to obtain the smallsignal acoustic temperature fluctuation, $\Delta T_{K}$ for an ideal adiabatic gas as

$$
\Delta T_{K}=\Delta p\left(\frac{\gamma-1}{\gamma}\right) \frac{T_{K 0}}{P_{0}} .
$$

Equation (11) will be the relationship we employ to relate/convert acoustic pressure to acoustic temperature. It is advantageous to use Eq. (11) because it relates the acoustic temperature measured by a temperature probe to the acoustic pressure measured by a microphone by two constants easily measured in the field. Also, at high amplitudes, the nonlinear relationship between pressure and temperature would necessitate the use of the shock adiabat at which point Eq. (9) would become invalid. The relative error between equations 9 and 11 is seen in the Fig.1a. From this Figure, we see that it is only as the acoustic pressure approaches $80 \%$ of $1 \mathrm{~atm}(80 \mathrm{kPa}$ or $19 \mathrm{X} \mathrm{dB}$ ) that the error between the methods becomes 1 decibel. Pressures variations of this magnitude induce temperature swings of more than 50 Kelvin. The plot in Fig. 1b shows how Eqs. (9) and (11) diverge when plotted on the same scale. The star located on the left side of the lower plot represents $T_{r e f}$, the reference temperature that will be used to later calculate the sound temperature level, STL, of the measured temperature data. $T_{\text {ref }}$ is found by using Eq. (11) and assuming the acoustic pressure to be that of an ideal plane wave propagating in air at $1 \mathrm{~atm}$ and $20^{\circ} \mathrm{C}$. For a plane wave with reference intensity $1 \mathrm{e}-12 \mathrm{~W} / \mathrm{m}^{\wedge} 2, T_{\text {ref }}$ has a value of $16.9 \mathrm{nK}$ which, as $20.4 \mu \mathrm{Pa}$ is rounded to $20 \mu \mathrm{Pa}$ for the reference pressure in determining SPL, the STL reference will be rounded to $17 \mathrm{nK}$. This temperature is the 
same temperature fluctuation derived if one starts with Eq. (4) and directly substitutes in the small signal relationship $\Delta p=c \Delta \rho$.

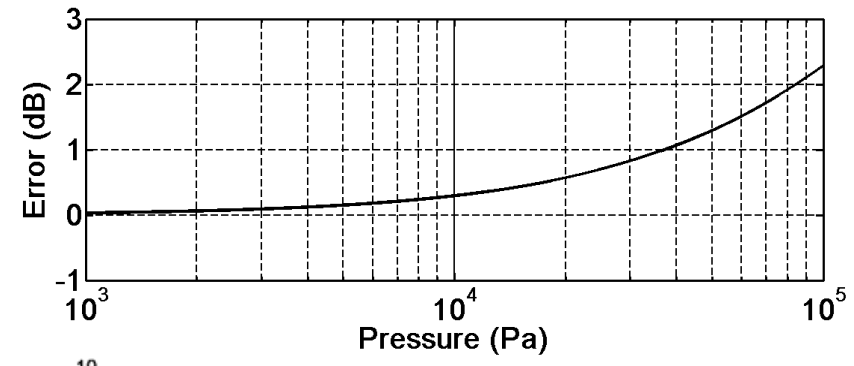

(a)

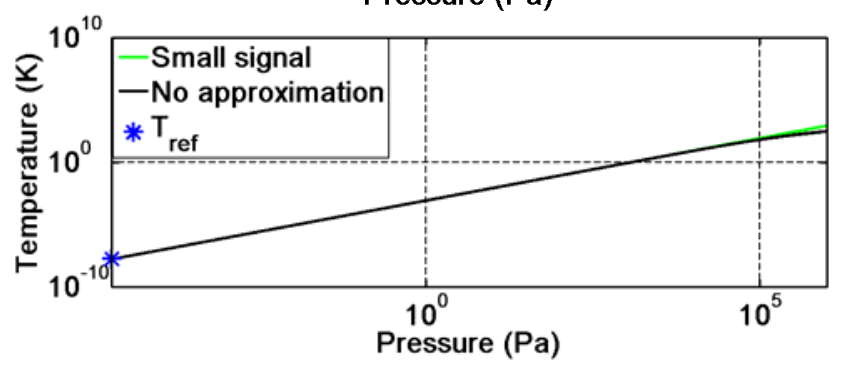

(b)

Fig. 1. (a) the error between equations 9 and 11 is shown. (b) The actual relationship between equations 9 and 11 . Note that $T_{r o f}$ is $17 \mathrm{nK}$.

\section{Plane wave tube}

The thin wire probe used in this experiment was a Dantec Dynamics 55P11 style general use probe. The probe is a straight configuration and consists of a single $.4 \mathrm{~mm}$ long platinum sensor, $1 \mu \mathrm{m}$ in diameter, spot welded to stainless steel prongs. Following the manufacturers advice, our configuration of this probe is quite typical of a CCA and it is powered by a $4-20 \mathrm{~mA}$ constant current supply which is connected to the probe via a 55H20 Dantec style probe support which was run through spec 55 twisted shielded pair wire. The voltage across the probe was measured using a NI PXI-4462 card set in a NI PXI-1040Q chassis.

Laboratory experiments were performed using the shock/plane wave tube to compare a 3.8-mm G.R.A.S. 40DP microphone to the temperature probe. The plane wave tube is 
constructed of 2" Schedule 40 PVC pipe with a 3-way ABS coupler attached to three 4591 BMS compression drivers connected in parallel and driven with the same acoustic input. The tube configuration is capable of producing peak pressures exceeding $10 \mathrm{kPa}$ (174 dB re $20 \mu \mathrm{Pa})$.

In an ideal plane wave tube, two sensors placed an equal distance down the tube should register the same magnitude and phase. This idea was employed to sample magnitude and phase relationship between discrete frequencies. The discrepancies of magnitude and phase between the two transducers were then compared with the transfer function result found using a switching technique calibration.

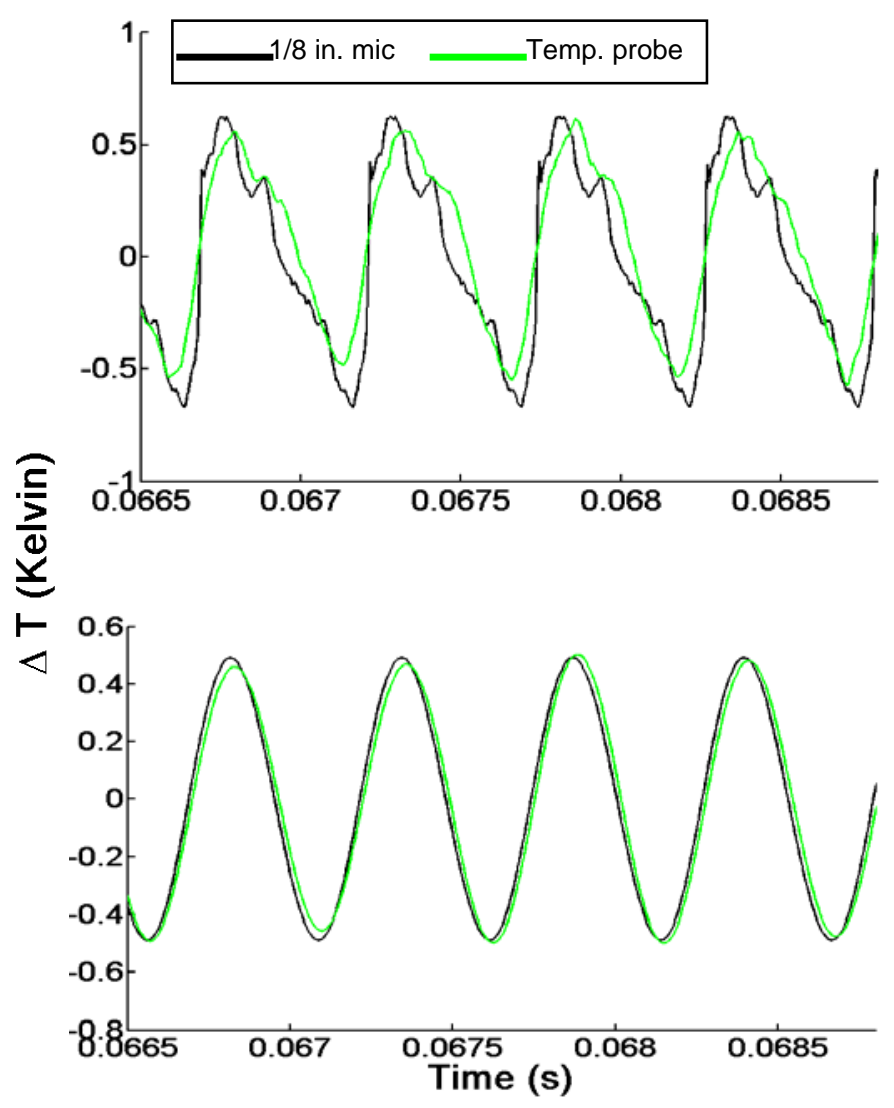

(a)

(b)

Fig. 2. Time data of a $1900 \mathrm{~Hz}$ sine wave in a plane/shock wave tube. (a) Unfiltered data of a steepened sine wave with harmonics generated. (b) Both transducers are band pass filtered $(500-2500 \mathrm{~Hz})$, noticeably eliminating the high frequency noise in the waveforms and improving the relationship between them. The high frequency noise of this steeped sine wave develops from small obstructions within the tube. Although aesthetically unpleasant, they do not degrade the usefulness of the data. 


\section{Rocket field}

\section{A. Setup}

Given the thermal lag of any temperature sensing device, it is advantageous to use large rocket motors for their extreme high amplitude and low frequency sound spectrum which allows for better resolving of the acoustic signal. In the desert of Northern Utah, ATK, a rocket development and testing facility, periodically tests full-scale rocket motors. One of these motors, the GEM-60, was the source for the temperature probes field test.

The GEM60 solid rocket motor has a 90 second burn time during which it has an average thrust of nearly 200,000 lbf. At our measurement location, this motor has a SPL of $160 \mathrm{~dB}$ re $20 \mu \mathrm{Pa}$ with the dominant energy around $100 \mathrm{~Hz}$. While these levels and frequency content are ideal for taking acoustic temperature measurements, radiant energy from burning aluminum particles in the plume and turbulence near the probe all but guarantee non-acoustic temperature fluctuations to be present. The relative importance of these error sources to our acoustic data is interesting for applications in fluid mechanics and acoustics. 


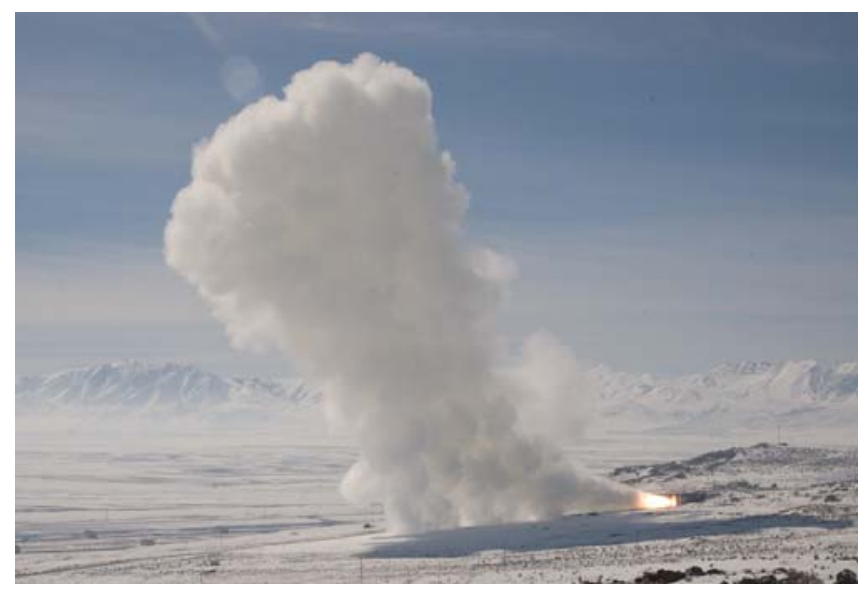

(a)

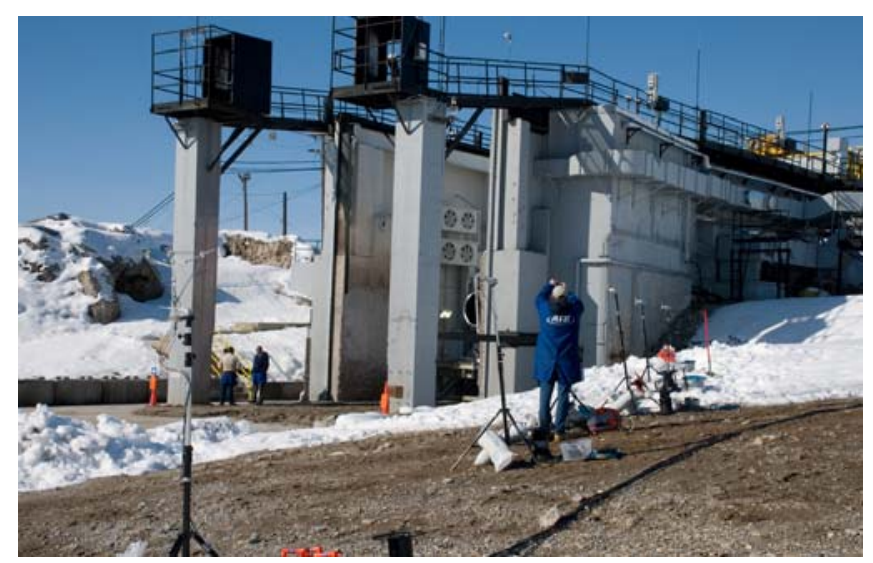

(b)

Fig. 3. (a) View of the GEM-60 Solid Rocket Motor during a static test. The brighter part of the plume is approx. 150 feet in length. (b) GEM-60 test bay with tripods aligned along the plume. The gentleman in the picture marks the location of the temperature probe and 1/8” microphone.

Both transducers were aligned along the angle of the plume with an offset to protect them from small particles and extreme temperatures (FIG. 3b). The in-field instrumentation setup was identical to that used in lab tests. Data collection was begun with a clock-based trigger approximately 5 min before the test at a sampling rate of 204800 samples/sec and continued beyond the end of the test. Two field tests were performed, one in June 2008 and another in February 2009. Both field tests were GEM60 solid rocket motors and were fired in the same test bay. The difference between the 
two tests was that the June test was a $90^{\circ} \mathrm{F}$ summer day and the February test was $40^{\circ} \mathrm{F}$ with snow on the ground, also, the June test included gimbaling of the rocket nozzle whereas in February the nozzle was stationary. Also, between tests, changes were made to the temperature probe instrumentation in an effort to increase bandwidth.

\section{B. Results}

Measurements from the February field test give the time waveform data in Fig 4. In all plots, the microphone data have been converted from pressure to temperature by means of our small signal approximation in Eq. (11).

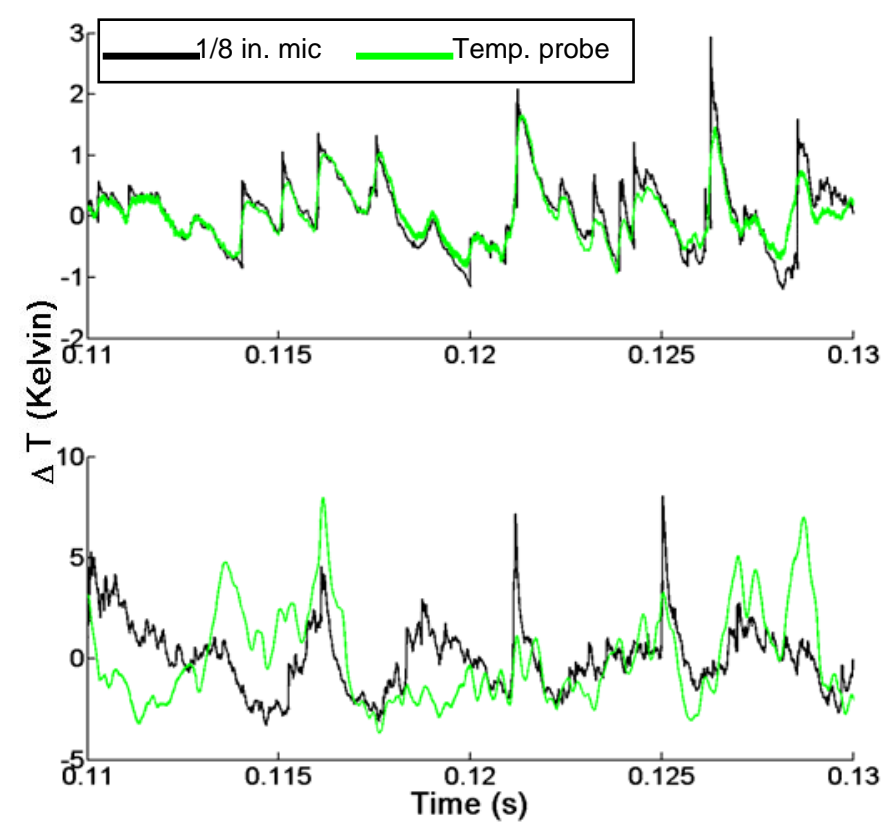

(a)

Fig. 4. Both plots show the temperature fluctuation of two transducers. (a) is a snapshot at the very beginning of the firing and shows most temperature fluctuations to be acoustic. (b) shows a similar time window 20 seconds into the test and non-acoustic temperature fluctuations are more important.

The temperature probe is recording data similar to that of the microphone with some discrepancies. The major difference between the two sensors is that temperature probe 
exhibits thermal lag and with its limited bandwidth is not able to capture the highfrequency content of the shocks. As time progresses further into the 90-second test firing, we can see (Fig. 4) that the relationship between the microphone and temperature probe begins to deteriorate. Some features are preserved between the transducers, but non-acoustic affects almost seem to dominate in the middle of the test.

The relative importance of nonacoustic temperature fluctuations can be observed by calculating the coherence between the microphone and temperature probe as a function of burn time. An interesting analysis of the coherence between the pressure microphone and temperature probe reveals that there is greater phase correlation between the transducers at the beginning of the test than there is throughout the firing. Fig 5 shows the coherence over frequency and time for the two GEM-60 tests.

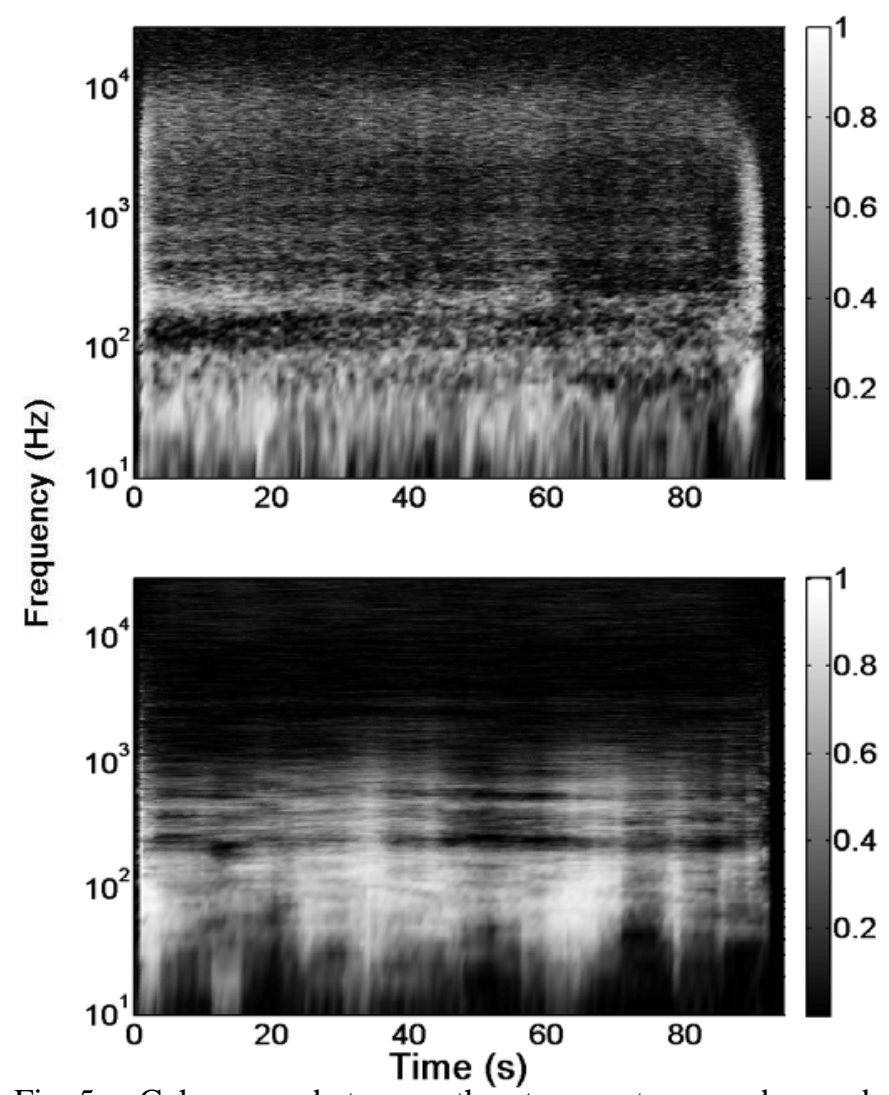

(a)

(b)

Fig. 5. Coherence between the temperature probe and microphone for two separate tests. It (a) Feb test on snowcovered ground. (b) June test on dry ground. Note the ground reflection around $100 \mathrm{~Hz}$, it is wider for the snowcovered ground of the February test The June test appears to have better low frequency coherence which could be partially due to less convective currents from the lack of snow. 
The June test shows greater coherence below $1000 \mathrm{hz}$ than does the February firing. It is the belief of the authors that the snow covered ground in the February test introduced a more thermally unpredictable environment and turbulent affects were more prominent at low frequencies. It is interesting to note that both tests had better coherence over frequency at the beginning of the test. This is most likely because the initial sound of the motor arrives before the turbulence reaches the probe setup.

Comparing the sound temperature level (STL) spectra at three different times for the February test, we again see the importance of turbulence during the test (FIG. 6). The spectra show the high frequency limit of the probe as an acoustic sensor particularly plots (a) and (b). Plot (b) also shows the impact of turbulence and other low frequency temperature fluctuations. Similar results are found for the June test.

It is important to note that while the coherence of the February test is good at the end of the test, the two transducers appeared to be $180^{\circ}$ degrees out of phase. Slight changes where made in instrumentation between the tests which might explain why this phase inversion did not occur during the June test, however, at this time we have no explanation why it occurred and lab test have flailed to reproduce the phenomenon. 


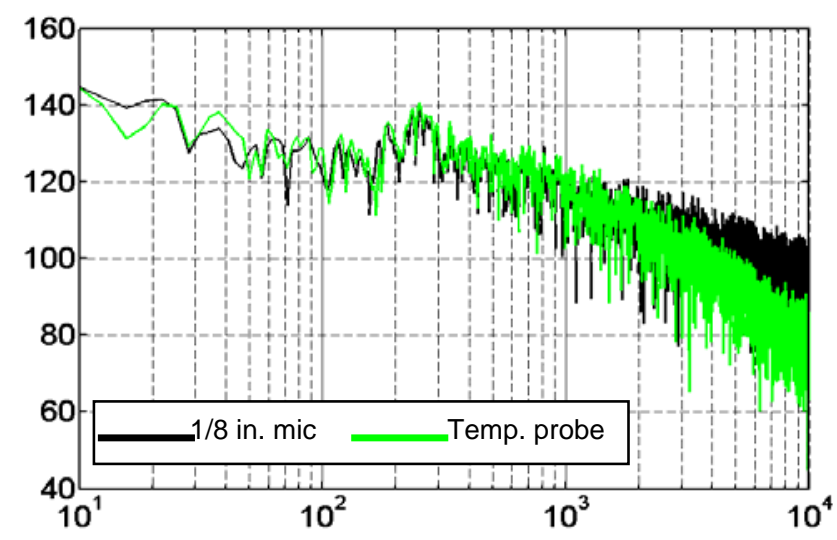

(a)

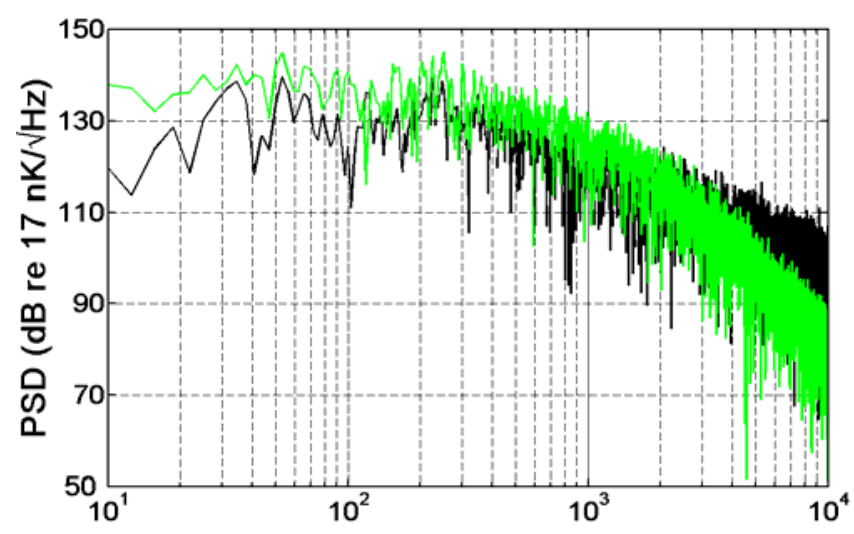

(b)

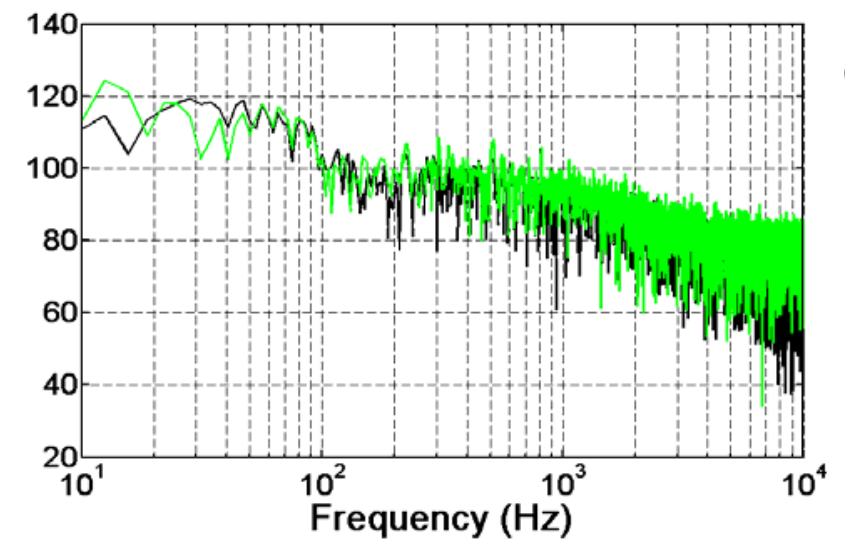

(c)

Fig. 6. These acoustic temperature level spectra show the difference between acoustic pressure and acoustic temperature measurements at (a) start of test, (b) 20 seconds into test, and (c) end of test. 


\section{Conclusion}

With the development of thin wire anemometry, past researchers have developed thin wire probes which can be used to take acoustic temperature measurements. It was found that thin wire transducers can be relatively calibrated using plane wave tube techniques. In future work, a thinner wire might be employed to extend the bandwidth of these temperature probes, however, the robustness of a thicker wire may be necessary in environments such as a rocket plume.

A relationship relating acoustic pressure and acoustic temperature was developed and a reference of $17 \mathrm{nK}$ has been presented for describing the sound temperature level of a noise source. The developed equation (11) was shown to be theoretically accurate for most acoustic measurements and was verified experimentally for noise at the beginning of full-scale solid rocket motor firing.

Coherence of the temperature probe and microphone was much better during the test in June which suggests that the snow likely played a role in emphasizing the non-acoustic temperature fluctuations in the measurements. It is also likely that the biggest factor influencing the accuracy of the time domain measurements was turbulence. The radiation from the rocket could also introduce error, but given the relative speeds of light and sound, the radiation should have made a noticeable impact at the beginning of the test as well as during the test. We are unable to definitively comment on the import of each error source, however, it has been shown that although muddled, the acoustic temperature fluctuations are measurable and important in a rocket field. 
The thin wire probe is capable of taking accurate acoustic measurements, however, with constant improvement to pressure transducers, including reduction in size, it is difficult to see the thin wire probe replacing traditional acoustic sensors. The future research of others, including that of this group, may find a use for such a transducer, but for now, the temperature probe appears to be a solution looking for a problem.

\section{Acknowledgements}

We would like to acknowledge Derek C. Thomas for his interest in and organization of this project and John E. Ellsworth for expertise in building the temperature probe's signal conditioning unit. 


\section{References}

${ }^{1}$ G. R. Ochs, "A resistance thermometer for measurement of rapid air temperature fluctuations,” ESSA Technical report, IER 47-ITSA 46, October 1967.

${ }^{2}$ N. E. J. Boston, and E. L. Sipe, “A high frequency platinum resistance thermometer system for measuring turbulent atmospheric temperature fluctuations," Naval Postgraduate School Technical Report, Period 1, July 1974-February 1975, February 1975.

${ }^{3}$ J. C. LaRue, T. Deaton, and C. H. Gibson, "Measurement of high-frequency turbulent temperature,” Review of Scientific Instruments, Vol. 46, No. 6, June 1975, pp. 757-764.

${ }^{4}$ G. Huelsz, and E. Ramos, “Temperature measurements inside the oscillatory boundary layer produced by acoustic waves,” Journal of the Acoustical Society of America, Vol. 103, No. 3, March 1998, pp. 1532-1537.

${ }^{5}$ G. Braissulis, A. Honkan, J. Andreopoulos, and C.B. Watkins, "Application of hot-wire anemometry in shock-tube flows," Experiments in Fluids, Vol. 19, No.1, 1995, pp. 29-37.

${ }^{6}$ J. Højstrup, K. Rasmussen, and S. E. Larsen, "Dynamic calibration of temperature wires in still air,” DISA Information, No. 20, September 1976, pp. 22-30. 
${ }^{7}$ L. E. Kinsler, A. R. Frey, A. B. Coppens, and J. V. Sanders, Fundamentals of Acoustics, Wiley, New York, 2000. 